\title{
CONTINUOUSLY TRANSLATING VECTOR-VALUED MEASURES BY
}

\author{
U. B. TEWARI AND M. DUTTA
}

\begin{abstract}
Let $G$ be a locally compact group and $A$ an arbitrary Banach space. $L^{p}(G, A)$ will denote the space of $p$-integrable $A$-valued functions on $G . M(G, A)$ will denote the space of regular $A$-valued Borel measures of bounded variation on $G$. In this paper, we characterise the relatively compact subsets of $L^{P}(G, A)$. Using this result, we prove that if $\mu \in M(G, A)$, such that either $x \rightarrow \mu_{x}$ or $x \rightarrow_{x} \mu$ is continuous, then $\mu \in L^{1}(G, A)$.
\end{abstract}

1. Introduction. Let $G$ be a locally compact group and let $\lambda$ be the left Haar measure on $G$. Let $A$ be an arbitrary Banach space. The space of $A$-valued regular Borel measures of bounded variation on $G$ will be denoted by $M(G, A)$. The space of $A$-valued $p$-integrable (see $\$ 2$ for proper definition) functions on $G$ will be denoted by $L^{p}(G, A)(1 \leqslant p<\infty)$. For $A=C$, the complex field, these spaces will be denoted by $M(G)$ and $L^{p}(G)$ respectively. It is a well-known result that if $\mu \in M(G)$ and either of the functions $x \rightarrow_{x} \mu$ and $x \rightarrow \mu_{x}$ is continuous, then $\mu \in L^{1}(G)$ ( $\$ 19.27$ of [2]). For the vector-valued case, similar arguments lead to the result that under these conditions $\mu$ is absolutely continuous with respect to $\lambda$ (Lemma 1 of $\$ 4$ ). However, in the vector-valued case, a measure absolutely continuous with respect to $\lambda$ need not be in $L^{1}(G, A)$ (for example, see [6]). Hence it is of interest to see whether under such conditions we can claim that $\mu \in$ $L^{1}(G, A)$. In Theorem 4 of $\S 4$ we prove that if either $x \rightarrow_{x} \mu$ or $x \rightarrow \mu_{x}$ is continuous then $\mu \in L^{1}(G, A)$. In proving this result we use the results of $\S 3$ where we characterise the relatively compact subsets of $L^{p}(G, A)$. For $L^{p}(G)$ this was done by Weil in [7].

2. Definitions and preliminaries. An $A$-valued function $F$ on $G$ is called countably valued if there exists a sequence of disjoint Borel sets $\left\{E_{i}\right\}_{i=1}^{\infty}$ such that $F$ is constant on each $E_{i}$ and is zero on $G \backslash \cup_{i=1}^{\infty} E_{i}$. Let $\nu$ be a nonnegative Borel measure. $F$ is called $\nu$-measurable if there exists a sequence of countably valued functions converging to $F$ a.e. $(\nu) . F$ is called weakly measurable if $\phi_{0} F$ is measurable for every $\phi \in A^{*}$, the dual of $A$. It can be shown that $F$ is $\nu$-measurable if and only if $F$ is weakly measurable and there exists a set $E \subset G$ with $\nu(E)=0$, such that $F(G \backslash E)$ is separable. $F$ is called measurable if $F$ is $\nu$-measurable for any positive Borel measure $\nu$. Thus $F$ is measurable if and only if $F$ is weakly measurable and has separable range. Two $\nu$-measurable functions equal a.e. ( $\nu)$ are called $\nu$-equivalent.

Received by the editors September 6, 1978.

AMS (MOS) subject classifications (1970). Primary 22D99, 28A45, 46G10.

Key words and phrases. Locally compact group, vector-valued measures. 
For $1<p<\infty, L^{p}(G, A)$ is the set of $\lambda$-equivalence classes of $\lambda$-measurable functions such that if $F$ is a representative of an equivalence class belonging to $L^{p}(G, A)$, then $\left(\int_{G}\|F\|^{p} d \lambda\right)^{1 / p}=\|F\|_{p}<\infty . L^{p}(G, A)$ with the norm $\|\cdot\| \|_{p}$ forms a Banach space. As usual, by a function in $L^{p}(G, A)$, we shall mean the corresponding equivalence class. For any function $F$ on $G$ and any $x \in G,{ }_{x} F$ will denote the left translate of $F$ by $x$, defined by ${ }_{x} F(y)=F(x y)$. Similarly we define the right translate $F_{x}$ by $F_{x}(y)=F(y x)$. If $F \in L^{p}(G, A)$ then both ${ }_{x} F$ and $F_{x}$ belong to $L^{p}(G, A)$ for any $x \in G$. Moreover, \|\|$_{x} F\left\|_{p}=\right\| F \|_{p}$ and $\left\|F_{x}\right\|_{p}=$ $\left[\Delta\left(x^{-1}\right)\right]^{1 / p} \mid\|F\|_{p}$, where $\Delta$ is the modular function on $G$. It can also be proved that the map $x \rightarrow{ }_{x} F$ of $G$ into $L^{P}(G, A)$ is right uniformly continuous and that the map $x \rightarrow F_{x}$ is continuous.

If $F$ is $\lambda$-measurable and $\int_{E}\|F\| d \lambda<\infty$ for some measurable set $E$, then we can define an integral (Bochner) of $F$ over $E \int_{E} F(x) d \lambda(x)$ as an element of $A$ (see [1] and [3]). Using this integral, we can define the convolution of $g \in L^{1}(G)$ and $F \in L^{p}(G, A)$ by

$$
g_{*} F(x)=\int_{G} g(x y) F\left(y^{-1}\right) d \lambda(y)=\int_{G} g(y) F\left(y^{-1} x\right) d \lambda(y)
$$

for almost all $x \cdot g_{*} F$ so defined belongs to $L^{p}(G, A)$ and $\|g * F\|_{p}<\|g\|_{L^{1}} \mid\|F\|_{p}$. Also if $\Delta^{-1 / p^{\prime}} g \in L^{1}(G)\left(p^{\prime}=p /(p-1), p^{\prime}=\infty\right.$ if $\left.p=1\right)$ and $F \in L^{p}(G, A)$, then we can define

$$
\begin{aligned}
F * g(x) & =\int_{G} \Delta\left(y^{-1}\right) g(y) F\left(x y^{-1}\right) d \lambda(y) \\
& =\int_{G} g(y x) F\left(y^{-1}\right) \Delta\left(y^{-1}\right) d \lambda(y)
\end{aligned}
$$

for almost all $x . F * g \in L^{p}(G, A)$ and $\|F * g\|_{p} \leqslant\|F\|_{p}\left\|\Delta^{-1 / p^{\prime}} g\right\|_{L^{1}}$. If support of $g \subset K_{1}$ and support of $F \subset K_{2}$ then support of $g * F \subset K_{1} K_{2}$ and support of $F * g \subset K_{2} K_{1}$. The proofs of these facts are exactly similar to the case when $A$ is the complex field (see [2]).

Let $\mathscr{B}$ denote the family of Borel subsets of $G$. Let $\mu$ be a countably additive $A$-valued function on $\mathscr{B} . V(\mu)$ will denote the total variation of $\mu$. $V(\mu)$ is a positive Borel measure on $G . \mu$ is said to be of bounded variation if $V(\mu)$ is finite. $\mu$ is called regular if $V(\mu)$ is regular. $\mu$ is said to be absolutely continuous with respect to $\lambda$ if $V(\mu)$ is absolutely continuous with respect to $\lambda . M(G, A)$ will denote the space of regular $A$-valued Borel measures of bounded variation on $G . M(G, A)$ is a Banach space under the norm $\|\mu\|_{v}=V(\mu)(G)$. For $\mu \in M(G, A)$ and $x \in G,{ }_{x} \mu$ will denote the left $x$-translate of $\mu$, defined by ${ }_{x} \mu(E)=\mu(x E)$ for any $E \in \mathscr{B}$. We define the right $x$-translate by $\mu_{x}(E)=\Delta\left(x^{-1}\right) \mu(E x)$ for any $E \in \mathscr{B} .\left(\Delta\left(x^{-1}\right)\right.$ is introduced in the definition of $\mu_{x}$ so that for $\mu \in L^{1}(G, A)$ the two definitions of $\mu_{x}$ coincide.)

For $\mu \in M(G, A)$ and $\nu \in M(G)$ we can use the results of Chapter II of [4] to define $\mu \times \nu$ and $\nu \times \mu$, the products of the measures $\mu$ and $\nu . \mu \times \nu$ and $\nu \times \mu$ are $A$-valued regular Borel measures on $G \times G$, the Cartesian product of $G$ with itself. Using this and the results of Chapter IV of [4] we can define $\mu * \nu$ and $\nu * \mu$, the 
convolutions of the measures $\mu$ and $\nu . \mu * \nu \in M(G, A)$ and is given by $\mu * \nu(E)=$ $\mu \times \nu\left(E_{2}\right)$, where $E_{2}=\{(x, y) \in G \times G: x y \in E\}$. Also $\nu * \mu \in M(G, A)$ and $\nu * \mu(E)=\nu \times \mu\left(E_{2}\right)$. Since $\nu$ is scalar-valued, we can use Theorem III.1 of [4] to get $\mu \times \nu\left(E_{2}\right)=\int_{G} \phi_{E_{2}}(x) d \mu(x)$. Here $\phi_{E_{2}}(x)=\nu\left(\left(E_{2}\right)_{x}\right)$ and $\left(E_{2}\right)_{x}=\{y \in G$ : $\left.(x, y) \in E_{2}\right\}=x^{-1} E$. Thus we get,

$$
\mu * \nu(E)=\int_{G} \nu\left(x^{-1} E\right) d \mu(x)
$$

If $\mu(\mathscr{B})$, the range of the vector-valued measure $\mu$, is separable then using the same theorem we get $\mu \times \nu\left(E_{2}\right)=\int_{G} \psi_{E_{2}}(y) d \nu(y)$. Here $\psi_{E_{2}}(y)=\mu\left(\left(E_{2}\right)^{y}\right)$ and $\left(E_{2}\right)^{y}=$ $\left\{x \in G:(x, y) \in E_{2}\right\}=E y^{-1}$. Thus we get,

$$
\mu * \nu(E)=\int_{G} \mu\left(E y^{-1}\right) d \nu(y)
$$

Similarly we get,

$$
\nu * \mu(E)=\int_{G} \nu\left(E y^{-1}\right) d \mu(y) .
$$

Also, if $\mu(\Re)$ is separable then,

$$
\nu * \mu(E)=\int_{G} \mu\left(x^{-1} E\right) d \nu(x)
$$

We note that the $\mu$-integrability of the integrands in (1) and (3), and the $\nu$-integrability of the integrands in (2) and (4) are part of the conclusions of Theorem III.1 of [4]. These integrals are Bochner-type integrals and are discussed in §III.1 of [4] and also in [1] and [3].

REMARK. We can show that equation (2) is valid whenever the function $y \rightarrow$ $\mu\left(E y^{-1}\right)$ has separable range. This function is weakly measurable. Hence it is measurable whenever it has separable range. Also,

$$
\begin{aligned}
\int\left\|\mu\left(E y^{-1}\right)\right\| d V(\nu)(y) & \leqslant \int V(\mu)\left(E y^{-1}\right) d V(\nu)(y) \\
& =V(\mu) * V(\nu)(E) .
\end{aligned}
$$

Hence, by $\S$ III.1 of [4], $y \rightarrow \mu\left(E y^{-1}\right)$ is $\nu$-integrable and the right-hand side of equation (2) is well defined. Let $A^{*}$ be the dual of $A$. Then for any $\phi \in A^{*}$

$$
\phi\left(\int \mu\left(E y^{-1}\right) d \nu(y)\right)=\int \phi_{0} \mu\left(E y^{-1}\right) d \nu(y)=\left(\phi_{0} \mu\right) * \nu(E) .
$$

From the definitions it easily follows that $\phi_{0}(\mu * \nu)=\left(\phi_{0} \mu\right) * \nu$. Hence,

$$
\phi_{0}(\mu * \nu)(E)=\left(\phi_{0} \mu\right) * \nu(E)=\phi\left(\int \mu\left(E y^{-1}\right) d \nu(y)\right) \text {. }
$$

Since $\phi \in A^{*}$ is arbitrary, we see that equation (2) is valid. Similarly we can show that equation (4) is valid whenever the function $x \rightarrow \mu\left(x^{-1} E\right)$ has separable range. We shall make use of these facts in the proof of Theorem 4.

From the definitions it follows easily that for any $x \in G,{ }_{x}(\mu * \nu)={ }_{x} \mu * \nu$ and $(\mu * \nu)_{x}=\mu * \nu_{x}$. Also from Theorem IV.2(b) of [4] we have $V(\mu * \nu)<$ $V(\mu) * V(\nu)$. 
Any $F \in L^{1}(G, A)$ defines an element of $M(G, A)$ which we will denote by $F$ itself. This element is given by $F(E)=\int_{E} F(x) d \lambda(x)$ for any $E \in \mathscr{B}$. This correspondence gives an isometric imbedding of $L^{1}(G, A)$ in $M(G, A)$.

3. Relatively compact subsets of $L^{p}(G, A)$. We now prove a theorem which characterises the relatively compact subsets of $L^{p}(G, A)$ for $1<p<\infty$.

THEOREM 1. Subset $\mathscr{F}$ of $L^{p}(G, A)$ is relatively compact if and only if the following conditions are satisfied.

(1) $\mathscr{F}$ is norm bounded, i.e., there exists a constant $M>0$ such that for any $F \in \mathscr{F},\|F\|_{p} \leqslant M$.

(2) Given $\varepsilon>0$, there exists a compact set $K \subset G$ such that $\sup \left\{\int_{G \backslash K}\|F\|^{p} d \lambda\right.$ : $F \in \mathscr{F}\}<\varepsilon$.

(3) Given $\varepsilon>0$, there exists a neighbourhood $U$ of identity $e$ in $G$ such that $\sup \left\{\|\|_{a} F-F \|_{p}: a \in U, F \in \mathcal{F}\right\}<\varepsilon$.

(4) For each measurable relatively compact subset $E$ of $G$, the set $\left\{\int_{E} F(x) d \lambda(x)\right.$ : $F \in \mathscr{F}\}$ is relatively compact in $A$.

(Note that $\int_{E} F(x) d \lambda(x)$ is defined even for $p>1$, since by Hölder's inequality, $\left.\int_{E}\|F(x)\| d \lambda(x)<\|F\|_{p}[\lambda(E)]^{1 / p^{\prime}}\right)$.

Proof. The necessity of (1)-(3) follows easily from total boundedness of $\mathscr{F}$. For (4) it is enough to note that the mapping $F \rightarrow \int_{E} F(x) d \lambda(x)$ is continuous from $L^{p}(G, A)$ into $A$.

For sufficiency, we shall construct a $5 \varepsilon$-net in $\mathscr{F}$ for any $\varepsilon>0$. Choose a compact set $K$ for $\varepsilon^{p}$ as in (2) and a compact symmetric neighbourhood $U$ for $\varepsilon$ as in (3). Let $\chi_{K}$ be the characteristic function of $K$. Choose a continuous nonnegative function $g$ on $G$ supported in $U$ with $\int_{G} g d \lambda=1$. For $F \in \mathscr{F}$, let $F^{*}=\chi_{K} F$ and $F^{* *}=g * F^{*}$. Then $\left\|F-F^{*}\right\|_{p}=\left[\int_{G \backslash K}\|F\|^{p} d \lambda\right]^{1 / p}<\varepsilon$. Also,

$$
\begin{aligned}
\|g * F(x)-F(x)\| & =\left\|\int_{G} g(y) F\left(y^{-1} x\right) d \lambda(y)-\int_{G} g(y) F(x) d \lambda(y)\right\| \\
& <\int_{G}\left\|F\left(y^{-1} x\right)-F(x)\right\| g(y) d \lambda(y) \\
& <\left[\int_{G}\left\|\left(y^{-1} F-F\right)(x)\right\|^{p} g(y) d \lambda(y)\right]^{1 / p} .
\end{aligned}
$$

Note that $\int_{G} g d \lambda=1$. Thus,

$$
\begin{aligned}
\|g * F-F\|_{p} & <\left[\int_{G} d \lambda(x) \int_{G}\left\|\left(y^{-1} F-F\right)(x)\right\|^{p} g(y) d \lambda(y)\right]^{1 / p} \\
& =\left[\int_{U} g(y) d \lambda(y) \int_{G}\left\|_{y^{-1}} F-F\right\|^{p} d \lambda\right]^{1 / p} \\
& <\left[\varepsilon^{p} \int_{U} g(y) d \lambda(y)\right]^{1 / p}=\varepsilon
\end{aligned}
$$


Therefore,

$$
\begin{aligned}
\left\|F^{* *}-F\right\|_{p} & <\left\|g * F^{*}-g * F\right\|_{p}+\|g * F-F\|_{p} \\
& <\|g\|_{L^{\prime}}\left\|F^{*}-F\right\|_{p}+\varepsilon<2 \varepsilon .
\end{aligned}
$$

Let $\mathscr{F}^{* *}$ denote the family of functions $F^{* *}$ for $F \in \mathscr{F}$. In view of the above inequality, an $\varepsilon$-net in $\mathscr{F}^{* *}$ will give a $5 \varepsilon$-net in $\mathscr{F}$.

To obtain an $\varepsilon$-net in $\mathscr{F}^{* *}$, we first prove that $\mathscr{F}^{* *}$ is an equicontinuous family of functions. Suppose $\varepsilon_{1}>0$. Let $M_{0}=\sup _{y \in K}\left[\Delta\left(y^{-1}\right)\right]$. Choose a neighbourhood $V$ of $e$ in $G$ such that $\left\|_{a} g-g\right\|_{p^{\prime}} \leqslant \varepsilon_{1} / M M_{0}^{1 / p}$ for all $a \in V$. Then for any $F^{* *} \in \mathcal{F}^{* *}$, $a \in V$ and $x \in G$, we have

$$
\begin{aligned}
\left\|F^{* *}(a x)-F^{* *}(x)\right\| & =\left\|\int_{G}[g(a x y)-g(x y)] F^{*}\left(y^{-1}\right) d \lambda(y)\right\| \\
& \leqslant \int_{G}\left|\left(a_{a x} g-{ }_{x} g\right)(y)\right|\left\|F^{*}\left(y^{-1}\right)\right\| d \lambda(y) \\
& <\left\|_{a x} g-{ }_{x} g\right\|_{p^{\prime}}\left[\int_{G}\left\|F^{*}\left(y^{-1}\right)\right\|^{p} d \lambda(y)\right]^{1 / p} \\
& =\left\|_{x}\left({ }_{a} g-g\right)\right\|_{p^{\prime}}\left[\int_{K}\left\|F^{*}(y)\right\|^{p} \Delta\left(y^{-1}\right) d \lambda(y)\right]^{1 / p} \\
& <\left\|_{a} g-g\right\|_{p^{\prime}} M_{0}^{1 / p}\left[\int_{K}\left\|F^{*}(y)\right\|^{p} d \lambda(y)\right]^{1 / p} \\
& <\frac{\varepsilon_{1}}{M M_{0}^{1 / p}} M_{0}^{1 / p}\left\|F^{*}\right\|_{p}<\varepsilon_{1} .
\end{aligned}
$$

This proves equicontinuity of $\mathscr{F}^{* *}$. Now, we shall prove that for any $x \in G$, the set $\left\{F^{* *}(x): F \in \mathscr{F}\right\}$ is relatively compact in $A$. We shall construct a $3 \varepsilon_{2}$-net in this set for any $\varepsilon_{2}>0$. Consider the function $g$ which is positive and continuous on $G$ and supported in $U$. Let $M^{\prime}=\sup _{y \in U}\left[\Delta\left(y^{-1}\right)\right]$. Let $h^{\prime}=\sum_{i=1}^{n} \alpha_{i} \chi_{E_{i}}$, where $E_{i}$ 's are disjoint measurable relatively compact subsets of $U$, such that $\left\|h^{\prime}-g \Delta\right\|_{p^{\prime}}<$ $\varepsilon_{2} / M M_{0}^{1 / p} M^{\prime}$. Let $h=h^{\prime} \Delta^{-1}$. Then $\|h-g\|_{p^{\prime}}=\left\|\left(h^{\prime}-g \Delta\right) \Delta^{-1}\right\|_{p^{\prime}}<\varepsilon_{2} / M M_{0}^{1 / p}$. Now for any $x \in G$ and $F \in \mathscr{F}$,

$$
\begin{aligned}
\left\|g * F^{*}(x)-h_{*} F^{*}(x)\right\| & \leqslant \int_{G}|(g-h)(x y)|\left\|F^{*}\left(y^{-1}\right)\right\| d \lambda(y) \\
& <\left\|_{x}(g-h)\right\|_{p^{\prime}}\left[\int_{G}\left\|F^{*}\left(y^{-1}\right)\right\|^{p} d \lambda(y)\right]^{1 / p} \\
& =\|(g-h)\|_{p^{\prime}}\left[\int_{K}\left\|F^{*}(y)\right\|^{p} \Delta\left(y^{-1}\right) d \lambda(y)\right]^{1 / p} \\
& <\frac{\varepsilon_{2}}{M M_{0}^{1 / p}} M_{0}^{1 / p}\left[\int_{K}\left\|F^{*}(y)\right\|^{p} d \lambda(y)\right]^{1 / p} \\
& =\varepsilon_{2}\left\|F^{*}\right\|_{p} / M<\varepsilon_{2} .
\end{aligned}
$$


In view of this inequality, any $\varepsilon_{2}$-net in the set $\left\{h_{*} F^{*}(x): F \in \mathscr{F}\right\}$ will give a $3 \varepsilon_{2}$-net in $\left\{F^{* *}(x): F \in \mathscr{F}\right\}$. Now,

$$
\begin{aligned}
h_{*} F^{*}(x) & =\sum_{i=1}^{n} \alpha_{i}\left(\chi_{E_{i}} \Delta^{-1}\right) * F^{*}(x) \\
& =\sum_{i=1}^{n} \alpha_{i} \int_{G}\left(\chi_{E_{i}} \Delta^{-1}\right)(x y) F^{*}\left(y^{-1}\right) d \lambda(y) \\
& =\sum_{i=1}^{n} \alpha_{i} \int_{G}\left(\chi_{E_{i}} \Delta^{-1}\right)\left(x y^{-1}\right) F^{*}(y) \Delta\left(y^{-1}\right) d \lambda(y) \\
& =\sum_{i=1}^{n} \alpha_{i} \Delta^{-1}(x) \int_{G} \chi_{E_{i}}\left(x y^{-1}\right) F^{*}(y) d \lambda(y) \\
& =\sum_{i=1}^{n} \alpha_{i} \Delta^{-1}(x) \int_{E_{i}^{-1} x \cap K} F(y) d \lambda(y) .
\end{aligned}
$$

By (4), the sets $\left\{\int_{E_{i}^{-1} x \cap K} F(y) d \lambda(y): F \in \mathscr{F}\right\}$ are relatively compact for $1<i<n$, and hence it follows that the set $\left\{h_{*} F^{*}(x): F \in \mathscr{F}\right\}$ is relatively compact in $A$. Thus we can construct an $\varepsilon_{2}$-net in this set and from this we will get a $3 \varepsilon_{2}$-net in $\left\{F^{* *}(x): F \in \mathscr{F}\right\}$. This proves that $\left\{F^{* *}(x): F^{* *} \in \mathscr{F}^{* *}\right\}$ is relatively compact in $A$ for any $x \in G$.

We note that the family of functions $\mathscr{F}^{* *}$ is supported in the compact set $U K$. Considering $\mathscr{F}^{* *}$ as a family of continuous functions from $U K$ into $A$, we see that this family satisfies the hypothesis of Theorem 7.17 of [5] (Ascoli's theorem). Hence it is relatively compact in the topology of uniform convergence on $U K$, i.e. in the supremum norm. Now an $\varepsilon[\lambda(U K)]^{-1 / p}$-net in this norm will give an $\varepsilon$-net in $\mathcal{F}^{* *}$ with the $\|\cdot\| \|_{p}$ norm. As we have already proved, this gives a $5 \varepsilon$-net in $\mathscr{F}$. Since $\varepsilon>0$ is arbitrary, we have proved that $\mathscr{F}$ is relatively compact. This completes the proof.

For $A=C$, the complex field, condition (4) is redundant and we get Weil's theorem [7]. This is true for finite dimensional spaces also. Condition (4) is important whenever $A$ is infinite dimensional. Indeed, whenever $A$ is infinite dimensional, the following is an example of a family $\mathscr{F} \subset L^{p}(G, A)$ satisfying (1)-(3) but not (4).

Take $B \subset A$ such that $B$ is bounded but not relatively compact. Take $f \in$ $L^{p}(G), f \neq 0$. Now define $\mathscr{F}=\{a f: a \in B\}$.

Condition (3) is the left equicontinuity of the functions in $\mathscr{F}$. A similar theorem can be proved with left equicontinuity replaced by right equicontinuity.

THEOREM 2. A subset $\mathscr{F}$ of $L^{P}(G, A)$ is relatively compact if and only if $\mathscr{F}$ satisfies conditions (1), (2) and (4) of Theorem 1, and the following condition.

(3)' Given $\varepsilon>0$, there exists a neighbourhood $U$ of identity $e$ in $G$ such that $\sup \left\{\left\|F_{a}-F\right\|_{p}: a \in U, F \in \mathscr{F}\right\}<\varepsilon$.

The proof of Theorem 2 is similar to that of Theorem 1. One has to take $F^{* *}=F * g$ in place of $F^{* *}=g_{*} F^{*}$ and $\int_{G} \Delta^{-1} g d \lambda=1$ in place of $\int_{G} g d \lambda=1$. Similar changes have to be made in the definition of $h$. We omit the details. 
If we demand both right and left equicontinuity then we can show that condition (1) follows from the rest. In other words, we shall prove

TheOREM 3. A subset $\mathscr{F}$ of $L^{p}(G, A)$ is relatively compact if and only if the following conditions are satisfied.

(1) Given $\varepsilon>0$, there exists a compact set $K \subset G$ such that $\sup \left\{\int_{G \backslash K}\|F\|^{p} d \lambda\right.$ : $F \in \mathcal{F}\}<\varepsilon$.

(2) Given $\varepsilon>0$, there exists a neighbourhood $U$ of identity $e$ in $G$ such that $\sup \left\{\|\|_{a} F-F\left|\left\|_{p},\right\| F_{a}-F\right| \|_{p}: a \in U, F \in \mathscr{F}\right\}<\varepsilon$.

(3) For each measurable relatively compact subset $E$ of $G$, the set $\left\{\int_{E} F(x) d \lambda(x)\right.$ : $F \in \mathcal{F}\}$ is relatively compact in $A$.

Proof. The necessity of the conditions is obvious. For sufficiency, in view of Theorem 1, it is enough to prove that (1)-(3) imply that $\sup \left\{\|F\| \|_{p}: F \in \mathscr{F}\right\}=M$ $<\infty$. For $\varepsilon=1$, choose a compact set $K \subset G$ as in (1) and a compact neighbourhood $U$ of $e$ in $G$ as in (2). Choose $\left\{x_{i}\right\}_{i=1}^{n} \subset K$ such that $\left\{U x_{i}\right\}_{i=1}^{n}$ is a cover of $K$. Let $F \in \mathscr{F}$ and

$$
F^{\prime}(x)=\frac{1}{\lambda(U)} \int_{x U} F(y) d \lambda(y)
$$

Then

$$
\left(F^{\prime}-F\right)(x)=\frac{1}{\lambda(U)} \int_{x U}(F(y)-F(x)) d \lambda(y)
$$

Therefore,

$$
\begin{aligned}
\left\|\left(F^{\prime}-F\right)(x)\right\| & <\frac{1}{\lambda(U)} \int_{x U}\|F(y)-F(x)\| d \lambda(y) \\
& =\frac{1}{\lambda(U)} \int_{U}\|F(x y)-F(x)\| d \lambda(y) \\
& <\left[\frac{1}{\lambda(U)} \int_{U}\|F(x y)-F(x)\|^{p} d \lambda(y)\right]^{1 / p} .
\end{aligned}
$$

Hence,

$$
\begin{aligned}
\int_{K}\left\|F^{\prime}-F\right\|^{p} d \lambda & <\frac{1}{\lambda(U)} \int_{K} d \lambda(x) \int_{U}\|F(x y)-F(x)\|^{p} d \lambda(y) \\
& =\frac{1}{\lambda(U)} \int_{U} d \lambda(y) \int_{K}\|F(x y)-F(x)\|^{p} d \lambda(x) \\
& <\frac{1}{\lambda(U)} \int_{U}\left\|F_{y}-F\right\|_{p}^{p} d \lambda(y) \\
& <1 .
\end{aligned}
$$


Also, for any $a \in U$ and any $x \in G$, we have

$$
\begin{aligned}
\left\|F^{\prime}(a x)-F^{\prime}(x)\right\| & =\left\|\frac{1}{\lambda(U)} \int_{a x U} F(y) d \lambda(y)-\frac{1}{\lambda(U)} \int_{x U} F(y) d \lambda(y)\right\| \\
& =\left\|\frac{1}{\lambda(U)} \int_{x U} F(a y) d \lambda(y)-\frac{1}{\lambda(U)} \int_{x U} F(y) d \lambda(y)\right\| \\
& <\frac{1}{\lambda(U)} \int_{x U}\left\|_{a} F-F\right\| d \lambda \\
& <\left[\frac{1}{\lambda(U)} \int_{x U}\left\|_{a} F-F\right\|^{p} d \lambda\right]^{1 / p} \\
& <\left[\frac{1}{\lambda(U)}\right]^{1 / p}=\alpha \text { (say). }
\end{aligned}
$$

Now $\left\{(1 / \lambda(U)) \int_{x U} \phi d \lambda: \phi \in \mathscr{F}\right\}$ is relatively compact. Therefore

$$
\sup \left\{\left\|(1 / \lambda(U)) \int_{x U} \phi d \lambda\right\|: \phi \in \mathcal{F}\right\}<\infty .
$$

Let

$$
N=\max _{1<i<n} \sup \left\{\left\|(1 / \lambda(U)) \int_{x_{i} U} \phi d \lambda\right\|: \phi \in \mathcal{F}\right\} .
$$

Then $\left\|F^{\prime}\left(x_{i}\right)\right\| \leqslant N$ for $i=1,2, \ldots, n$. Since $U x_{i}$ 's cover $K$, and $\left\|F^{\prime}\left(a x_{i}\right)-F^{\prime}\left(x_{i}\right)\right\|$ $<\alpha$ for all $a \in U$ and $1 \leqslant i<n$, we have $\left\|F^{\prime}(x)\right\|<N+\alpha$ for all $x \in K$. Therefore, $\int_{K}\left\|F^{\prime}\right\|^{p} d \lambda \leqslant(N+\alpha)^{p} \lambda(K)=\beta^{p}$ (say). But $\int_{K}\left\|F^{\prime}-F\right\|^{p} d \lambda<1$. Therefore, $\int_{K}\|F\|^{p} d \lambda<(\beta+1)^{p}$. Thus $\int_{G}\|F\|^{p} d \lambda<(\beta+1)^{p}+1$ and we can take $M=\left[(\beta+1)^{p}+1\right]^{1 / p}$. This proves the theorem.

4. Continuously translating elements of $M(G, A)$. We now prove that the elements of $L^{1}(G, A)$ are the only ones in $M(G, A)$ which translate continuously. More precisely we prove

Theorem 4. If $\mu \in M(G, A)$ is such that either $x \rightarrow_{x} \mu$ or $x \rightarrow \mu_{x}$ is continuous, then $\mu \in L^{1}(G, A)$.

Before proving the theorem we prove a couple of lemmas.

LemMA 1. Let $\mu \in M(G, A)$. Then the following are equivalent.

(1) $\mu$ is absolutely continuous with respect to $\lambda$.

(2) For any measurable relatively compact set $E \subset G$, the function $y \rightarrow \mu(E y)$ is continuous.

(3) For any measurable relatively compact set $E \subset G$, the function $y \rightarrow \mu(y E)$ is continuous.

Proof. Let $\mu \in M(G, A)$ be absolutely continuous with respect to $\lambda$ and let $E$ be any measurable relatively compact subset of $G$. Let $y_{0} \in G$ and let $\varepsilon>0$ be given. Choose $\delta>0$ such that for any $F \subset G, \lambda(F)<\delta$ implies $V(\mu)(F)<\varepsilon$. This is possible since $V(\mu)$ is absolutely continuous with respect to $\lambda$. Now, $\chi_{E} \in L^{1}(G)$ 
since $E$ is relatively compact. Hence $y \rightarrow \chi_{E y}$ is continuous and we can choose a neighbourhood $V$ of $y_{0}$ such that for any $y \in V,\left\|\chi_{E y}-\chi_{E y_{0}}\right\|_{L^{1}}<\delta$. Therefore, for any $y \in V$,

$$
\begin{aligned}
\lambda\left(E y \Delta E y_{0}\right) & =\lambda\left(E y \backslash E y_{0}\right)+\lambda\left(E y_{0} \backslash E y\right) \\
& =\left\|\chi_{E y}-\chi_{E y_{0}}\right\|_{L^{\prime}}<\delta .
\end{aligned}
$$

Hence, for any $y \in V$,

$$
\begin{aligned}
\left\|\mu(E y)-\mu\left(E y_{0}\right)\right\| & =\left\|\mu\left(E y \backslash E y_{0}\right)-\mu\left(E y_{0} \backslash E y\right)\right\| \\
& \leqslant V(\mu)\left(E y \backslash E y_{0}\right)+V(\mu)\left(E y_{0} \backslash E y\right) \\
& =V(\mu)\left(E y \Delta E y_{0}\right)<\varepsilon .
\end{aligned}
$$

This shows that $y \rightarrow \mu(E y)$ is continuous. This proves $(1) \Rightarrow(2)$. The proof of $(1) \Rightarrow(3)$ is similar.

For the proof of $(3) \Rightarrow(1)$, let $\mu \in M(G, A)$ such that (3) is satisfied. Let $E$ be any compact subset of $G$ such that $\lambda(E)=0$. Then the function $x \rightarrow \| \mu\left(x^{-1} E\right)_{\|}^{\|}$is continuous. Let $\nu \in M(G)$ be defined by $d \nu=\chi_{U} d \lambda$, where $\chi_{U}$ is the characteristic function of some relatively compact neighbourhood $U$ of $e$. Then $\nu$ is absolutely continuous with respect to $\lambda$. Hence $\nu * V(\mu)$ is absolutely continuous with respect to $\lambda$. Therefore, we have

$$
\begin{aligned}
0 & =\nu * V(\mu)(E)=\int_{G} V(\mu)\left(x^{-1} E\right) d \nu(x) \\
& =\int_{U} V(\mu)\left(x^{-1} E\right) d \lambda(x) \geqslant \int_{U}\left\|\mu\left(x^{-1} E\right)\right\| d \lambda(x) .
\end{aligned}
$$

Since $x \rightarrow\left\|\mu\left(x^{-1} E\right)\right\|$ is a nonnegative continuous function, $\left\|\mu\left(x^{-1} E\right)\right\|=0$ for any $x \in$ Interior of $U$. Hence $\|\mu(E)\|=0$. In the same way $\|\mu(F)\|=0$ for any measurable $F \subset E$. Hence $V(\mu)(E)=0$. This shows that $V(\mu)$ is absolutely continuous with respect to $\lambda$. This proves $(3) \Rightarrow(1)$. The proof of $(2) \Rightarrow(1)$ is similar and the proof of Lemma 1 is complete.

Note. The proof of $(3) \Rightarrow(1)$ is an adaptation of $\$ 19.27$ of [2] to the vector-valued case.

LEMMA 2. Let $\mu \in M(G, A)$ and let $E$ be any measurable relatively compact subset of $G$. Then the functions $y \rightarrow \mu(E y)$ and $y \rightarrow \mu(y E)$ vanish at infinity.

Proof. Let $\varepsilon>0$ be given. By regularity of $\mu$ there exists a compact set $K \subset G$ such that $V(\mu)\left(K^{c}\right)<\varepsilon$ where $K^{c}$ is the complement of $K$. Let $K_{1}=E^{-1} K$. Then $K_{1}$ is relatively compact and for $y \notin K_{1}, E y \subset K^{c}$. Thus for $y \notin K_{1},\|\mu(E y)\|<\varepsilon$. This shows that the function $y \rightarrow \mu(E y)$ vanish at infinity. Similarly we can show that the function $y \rightarrow \mu(y E)$ also vanishes at infinity and our proof is complete.

Proof of Theorem 4. Let $\mu \in M(G, A)$ such that $x \rightarrow_{x} \mu$ is continuous. Then for any measurable set $E \subset G, x \rightarrow{ }_{x} \mu(E)=\mu(x E)$ is continuous. Hence by Lemmas 1 and 2 we can conclude that for any measurable relatively compact set $E$, the funtions $y \rightarrow \mu(y E), y \rightarrow \mu\left(y^{-1} E\right), y \rightarrow \mu(E y)$ and $y \rightarrow \mu\left(E y^{-1}\right)$ are continuous functions vanishing at infinity. 
We now take a fixed compact neighbourhood $U_{0}$ of the identity $e$ in $G$. Let $\mathscr{D}$ be the family of all neighbourhoods of $e$ contained in $U_{0}$ directed under inclusion. Take any $W \in \mathscr{Q}$. Then $\lambda(W)<\infty$ since $W$ is contained in the compact set $U_{0}$. Let $f_{W}=(1 / \lambda(W)) \chi_{W}$, where $\chi_{W}$ is the characteristic function of $W$. Then $f_{W} \in L^{1}(G)$ and $\left\|f_{W}\right\|_{L^{1}}=1$. Now $F_{W}=\mu * f_{W} \in M(G, A)$ and $\left\|F_{W}\right\|_{0}<\|\mu\|_{v}$. Let $\mathscr{F}=\left\{F_{W}: W \in \mathscr{D}\right\}$. We shall first prove that $\mathscr{F} \subset L^{1}(G, A)$. (See the remark at the end of this paper.) For this, take any $W \in \mathscr{D}$ and consider the $A$-valued function $F_{W}^{\prime}$ on $G$ defined by $F_{W}^{\prime}(y)=\int_{G} f_{W}\left(x^{-1} y\right) d \mu(x)=\mu\left(y W^{-1}\right) / \lambda(W)$. Since $W$, and hence $W^{-1}$, is relatively compact, it follows from the first paragraph of this proof that $F_{W}^{\prime}$ is a continuous function vanishing at infinity. Hence $F_{W}^{\prime}$ is measurable. Also

$$
\left\|F_{W}^{\prime}(y)\right\| \leqslant \int_{G} f_{W}\left(x^{-y}\right) d V(\mu)(x)=V(\mu) * f_{W}(y)
$$

Since $V(\mu) * f_{W} \in L^{1}(G)$ we see that $F_{W}^{\prime} \in L^{1}(G, A)$. Let $\phi$ be any element of $A^{*}$, the dual of $A$. Then for any $E \in \mathscr{B}$,

$$
\begin{aligned}
\phi\left(F_{W}^{\prime}(E)\right) & =\phi\left(\int_{E} F_{W}^{\prime}(y) d \lambda(y)\right)=\int_{E}\left(\phi_{0} F_{W}^{\prime}\right)(y) d \lambda(y) \\
& =\int_{E} d \lambda(y) \phi\left(\int_{G} f_{W}\left(x^{-1} y\right) d \mu(x)\right) \\
& =\int_{E} d \lambda(y) \int_{G} f_{W}\left(x^{-1} y\right) d\left(\phi_{0} \mu\right)(x) \\
& =\left(\phi_{0} \mu\right) * f_{W}(E) \\
& =\int_{G} f_{W}\left(x^{-1} E\right) d\left(\phi_{0} \mu\right)(x) \\
& =\phi\left(\int_{G} f_{W}\left(x^{-1} E\right) d \mu(x)\right) \\
& \left.=\phi\left(F_{W}(E)\right) \quad \text { (by (1) of } \S 2\right) .
\end{aligned}
$$

Hence $F_{W}(E)=F_{W}^{\prime}(E)$ for any $E \in \mathscr{B}$. Therefore $F_{W}=F_{W}^{\prime}$ and thus $F_{W} \in$ $L^{1}(G, A)$. Since $W$ is an arbitrary member of $\mathscr{D}$ we see that $\mathscr{F} \subset L^{1}(G, A)$.

Now we shall prove that $\mathcal{F}$ as a subset of $L^{1}(G, A)$ satisfies conditions (1)-(4) of Theorem 1. Since for any $W \in \mathscr{D},\left\|F_{W}\right\|_{1}=\left\|F_{W}\right\|_{v}<\|\mu\|_{v}$, we see that (1) is satisfied with $M=\|\mu\|_{v}$. Next, let $\varepsilon>0$ be given. Choose a compact set $K_{1} \subset G$ such that $V(\mu)\left(K_{1}^{c}\right)<\varepsilon$. Let $K=K_{1} U_{0}$. Then $K$ is compact and for any $W \in \mathscr{D}$,

$$
\begin{aligned}
\int_{G \backslash K}\left\|F_{W}(x)\right\| d \lambda(x) & =V\left(F_{W}\right)\left(K^{c}\right) \leqslant V(\mu) * f_{W}\left(K^{c}\right) \\
& \leqslant \frac{1}{\lambda(W)} \int_{W} V(\mu)\left(K y^{-1}\right) d \lambda(y)
\end{aligned}
$$


Now for any $x \in K^{c}$ and for any $y \in W, x y^{-1} \in K_{1}^{c}$. Therefore for any $y \in W$, $K^{c} y^{-1} \subset K_{1}^{c}$ and thus $V(\mu)\left(K^{c} y^{-1}\right)<\varepsilon$. Hence

$$
\begin{aligned}
\int_{G \backslash K}\left\|F_{W}(x)\right\| d \lambda(x) & \leqslant \frac{1}{\lambda(W)} \int_{W} V(\mu)\left(K^{c} y^{-1}\right) d \lambda(y) \\
& \leqslant \frac{\varepsilon}{\lambda(W)} \int_{W} d \lambda(y)=\varepsilon .
\end{aligned}
$$

Thus $\mathscr{F}$ satisfies condition (2) of Theorem 1.

Again for $\varepsilon>0$, we take a neighbourhood $U$ of $e$ in $G$, such that for any $x \in U$, $\left\|_{x} \mu-\mu\right\|_{0}<\varepsilon$. This is possible since $x \rightarrow_{x} \mu$ is continuous. Then for any $W \in \mathscr{Q}$ and for any $x \in U$,

$$
\begin{aligned}
\|\|_{x} F_{W}-F_{W} \|_{1} & =\left\|_{x}\left(\mu * f_{W}\right)-\mu * f_{W}\right\|_{v} \\
& =\left\|_{x} \mu * f_{W}-\mu * f_{W}\right\|_{v}=\left\|\left({ }_{x} \mu-\mu\right) * f_{W}\right\|_{v} \leqslant\left\|_{x} \mu-\mu\right\|_{v}\left\|f_{W}\right\|_{L^{1}} \leqslant \varepsilon .
\end{aligned}
$$

Thus $\mathscr{F}$ satisfies condition (2) of Theorem 1.

Finally, let $E$ be any measurable relatively compact subset of $G$. We shall show that $\left\{F_{W}(E): W \in \mathscr{D}\right\}$ is relatively compact in $A$. First we note that since $E$ is relatively compact, the function $y \rightarrow \mu\left(E y^{-1}\right)$ is a continuous function vanishing at infinity. Thus this function has separable range. Hence by the remark in $\$ 2$, equation (2) is valid for $\mu$. Thus

$$
F_{W}(E)=\int_{G} \mu\left(E y^{-1}\right) d f_{W}(y)=\frac{1}{\lambda(W)} \int_{W} \mu\left(E y^{-1}\right) d \lambda(y) .
$$

Since $y \rightarrow \mu\left(E y^{-1}\right)$ is continuous and $U_{0}$ is compact the function $y \rightarrow \mu\left(E y^{-1}\right)$ is uniformly continuous on $U_{0}$, i.e., given $\varepsilon>0$, there exists a neighbourhood $W_{0}$ of $e$, such that for any $x, y \in U_{0}$ with $x y^{-1} \in W_{0},\left\|\mu\left(E x^{-1}\right)-\mu\left(E y^{-1}\right)\right\|<\varepsilon$. Cover $U_{0}$ with finite number of right translates of $W_{0},\left\{W_{0} x_{i}\right\}_{i=1}^{n}$. Then any $W \in \mathscr{D}$ can be expressed as $W=\cup_{i=1}^{m} W_{i}$, where $W_{i}$ 's are disjoint measurable sets and each $W_{i} \subset W_{0} x_{k_{i}}$ for some $1<k_{i} \leqslant n$. Now if $x \in W_{i}$ then $x \in W_{0} x_{k_{i}}$ and hence $x x_{k_{i}}^{-1} \in W_{0}$. Thus for $x \in W_{i},\left\|\mu\left(E x_{k_{i}}^{-1}\right)-\mu\left(E x^{-1}\right)\right\|<\varepsilon$. Therefore,

$$
\begin{aligned}
\left\|F_{W}(E)-\sum_{i=1}^{m} \frac{\lambda\left(W_{i}\right) \mu\left(E x_{k_{i}}^{-1}\right)}{\lambda(W)}\right\| & \\
& =\frac{1}{\lambda(W)}\left\|\sum_{i=1}^{m} \int_{W_{i}} \mu\left(E x^{-1}\right) d \lambda(x)-\sum_{i=1}^{m} \lambda\left(W_{i}\right) \mu\left(E x_{k_{i}}^{-1}\right)\right\| \\
& <\frac{1}{\lambda(W)} \sum_{i=1}^{m} \int_{W_{i}}\left\|\mu\left(E x^{-1}\right)-\mu\left(E x_{k_{i}}^{-1}\right)\right\| d \lambda(x) \\
& <\frac{\varepsilon}{\lambda(W)} \sum_{i=1}^{m} \int_{W_{i}} d \lambda(x)=\varepsilon .
\end{aligned}
$$

Let $Y$ be the finite dimensional linear space generated by $\left\{\mu\left(E x_{j}^{-1}\right)\right\}_{j=1}^{n}$. Then we see that for any $W \in \mathscr{D}$, there exists $a_{W} \in Y$ such that $\left\|F_{W}(E)-a_{W}\right\|<\varepsilon$. Since 
$\left\{F_{W}(E): W \in \mathscr{D}\right\}$ is bounded, $\left\{a_{W}: W \in \mathscr{D}\right\}$ is a bounded subset of the finite dimensional linear space $Y$. Hence $\left\{a_{W}: W \in \mathscr{D}\right\}$ is totally bounded and we can obtain an $\varepsilon$-net $\left\{a_{W_{(0)}}\right\}_{i=1}^{m}$ in $\left\{a_{W}: W \in \mathscr{D}\right\}$. Then it is easy to see that $\left\{F_{W_{(0}}(E)\right\}_{i=1}^{m}$ is a $3 \varepsilon$-net in $\left\{F_{W}(E): W \in \mathscr{D}\right\}$. Since $\varepsilon$ is arbitrary, we can conclude that $\left\{F_{W}(E): W \in \mathscr{D}\right\}$ is totally bounded and hence relatively compact in $A$. This shows that $\mathscr{F}$ satisfies condition (4) of Theorem 1.

Thus $\mathscr{F}$ satisfies all the conditions of Theorem 1 and hence $\mathscr{F}$ is relatively compact as a subset of $L^{1}(G, A)$. Therefore the net $\left\{F_{W}: W \in \mathscr{D}\right\}$ has a subset which converges to some $F \in L^{1}(G, A)$. Let $E$ be any measurable relatively compact subset of $G$. Then the corresponding subnet of $\left\{F_{W}(E): W \in \mathscr{D}\right\}$ converges to $F(E)$. However since $E$ is relatively compact we have $F_{W}(E)=$ $(1 / \lambda(W)) \int_{W} \mu\left(E y^{-1}\right) d \lambda(y)$ as has been already shown. Since $E$ is relatively compact $y \rightarrow \mu\left(E y^{-1}\right)$ is continuous. Hence, given $\varepsilon>0$, we can choose a neighbourhood $U$ of $e$ in $G$ such that for any $y \in U,\left\|\mu\left(E y^{-1}\right)-\mu(E)\right\|<\varepsilon$. Then for $W \subset U$ and for $y \in W,\left\|\mu\left(E y^{-1}\right)-\mu(E)\right\|<\varepsilon$. Therefore for $W \in$ and $W \subset$ $U$,

$$
\begin{aligned}
\left\|F_{W}(E)-\mu(E)\right\| & \leqslant\left\|\frac{1}{\lambda(W)} \int_{W}\left\{\mu\left(E y^{-1}\right)-\mu(E)\right\} d \lambda(y)\right\| \\
& \leqslant \frac{1}{\lambda(W)} \int_{W}\left\|\mu\left(E y^{-1}\right)-\mu(E)\right\| d \lambda(y) \\
& \leqslant \frac{\varepsilon}{\lambda(W)} \int_{W} d \lambda(y)=\varepsilon .
\end{aligned}
$$

Therefore the net $\left\{F_{W}(E): W \in \mathscr{D}\right\}$ converges to $\mu(E)$. Hence any subnet of it also converges to $\mu(E)$ and thus $\mu(E)=F(E)$ for any measurable relatively compact subset $E$ of $G$. Since $\mu$ and $F$ are regular this equality remains valid for all measurable subsets $E$ of $G$. Thus $\mu=F \in L^{1}(G, A)$. This completes the proof of one-half of the theorem.

For the proof of the other half of the theorem, let $\mu \in M(G, A)$ such that $x \rightarrow \mu_{x}$ is continuous. Then for any measurable set $E \subset G, x \rightarrow \mu_{x}(E)=\Delta\left(x^{-1}\right) \mu(E x)$ is continuous. However $x \rightarrow \Delta(x)$ is continuous. Therefore, $x \rightarrow \mu(E x)$ is continuous. Hence by Lemmas 1 and 2 we can conclude that for any measurable relatively compact set $E$, the functions $y \rightarrow \mu(y E), y \rightarrow \mu\left(y^{-1} E\right), y \rightarrow \mu(E y)$ and $y \rightarrow$ $\mu\left(E y^{-1}\right)$ are continuous functions vanishing at infinity.

The rest of the proof is similar to that of the first half of the theorem. Instead of $F_{W}=\mu * f_{W}$ we shall have to take $F_{W}=f_{W} * \mu$. As before, we shall be able to prove that $\mathscr{F}=\left\{F_{W}: W \in \mathscr{D}\right\} \subset L^{1}(G, A)$. Theorem 2, instead of Theorem 1, will be used to prove that $\mathscr{F}$ is relatively compact in $L^{1}(G, A)$ and as before we will be able to conclude that $\mu \in L^{1}(G, A)$. This completes the proof.

Remark. We feel that there should be a direct proof of the fact that $\mathscr{F} \subset$ $L^{1}(G, A)$. Indeed from Theorem 4 it can be deduced that for any $\mu \in M(G, A)$ and $f \in L^{1}(G), \mu * f \in L^{1}(G, A)$. This is because $x \rightarrow(\mu * f)_{x}=\mu * f_{x}$ is continuous. It will be interesting to have a direct proof of this fact. 


\section{REFERENCES}

1. N. Dinculeanu, Vector measures, Pergamon, Oxford and New York, 1967.

2. E. Hewitt and K. A. Ross, Abstract harmonic analysis. I, Springer-Verlag, Berlin and New York, 1963.

3. E. Hille and R. S. Phillips, Functional analysis and semigroups, Amer. Math. Soc. Colloq. Publ., vol. 31, Amer. Math. Soc., Providence, R. I., 1957.

4. J. E. Huneycutt Jr., Products and convolutions of vector-valued set functions, Studia Math. 41 (1972), 101-129.

5. J. L. Kelley, General topology, Van Nostrand, Princeton, N. J., 1955.

6. J. J. Uhl, Jr., The range of a vector-valued measure, Proc. Amer. Math. Soc. 23 (1969), 158-163.

7. A. Weil, L'integration dans les groupes topologiques et ses applications, Hermann, Paris, 1951.

Department of Mathematics, Indin Institute of Technology, KanpUr-208016, India (Current address of U. B. Tewari)

Current address (M. Dutta): Quarter No. 108, Gauhati University, Gauhati, India 